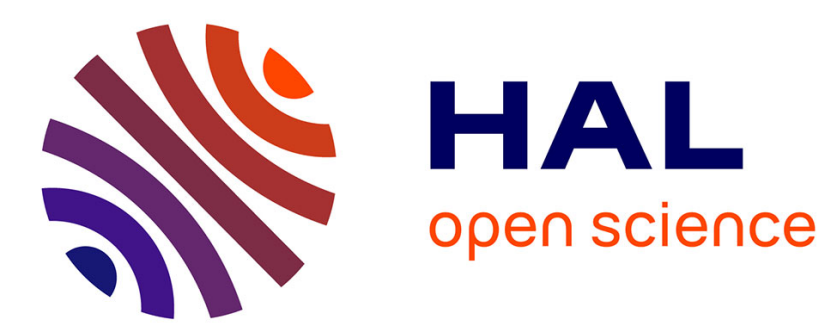

\title{
Les variables psychologiques médiatisées par le serious game dans l'insertion et la formation
}

\author{
Mariama Lobsang Ndao, Daniel Gilibert, Jérôme Dinet
}

\section{To cite this version:}

Mariama Lobsang Ndao, Daniel Gilibert, Jérôme Dinet. Les variables psychologiques médiatisées par le serious game dans l'insertion et la formation. Revue internationale de psychosociologie et de gestion des comportements organisationnels, 2017, XXIII (55), pp.277-296. 10.3917/rips1.055.0277 . hal-01783438

\section{HAL Id: hal-01783438 \\ https://hal.science/hal-01783438}

Submitted on 12 Oct 2018

HAL is a multi-disciplinary open access archive for the deposit and dissemination of scientific research documents, whether they are published or not. The documents may come from teaching and research institutions in France or abroad, or from public or private research centers.
L'archive ouverte pluridisciplinaire HAL, est destinée au dépôt et à la diffusion de documents scientifiques de niveau recherche, publiés ou non, émanant des établissements d'enseignement et de recherche français ou étrangers, des laboratoires publics ou privés. 


\section{LES VARIABLES PSYCHOLOGIQUES MÉDIATISÉES PAR LE SERIOUS GAME DANS L'INSERTION ET LA FORMATION}

Mariama Lobsang Ndao, Daniel Gilibert, Jérôme Dinet

\section{ESKA | « Revue internationale de psychosociologie et de gestion des comportements} organisationnels »

2017/1 Vol. XXIII | pages 277 à 296

ISSN 2262-8401

ISBN 9782747226882

\section{Article disponible en ligne à l'adresse :}

https://www.cairn.info/revue-internationale-de-psychosociologie-de-gestion-descomportements-organisationnels-2017-1-page-277.htm

\section{Pour citer cet article :}

Mariama Lobsang Ndao et al., « Les variables psychologiques médiatisées par le serious game dans l'insertion et la formation », Revue internationale de psychosociologie et de gestion des comportements organisationnels 2017/1 (Vol. XXIII), p. 277-296.

Distribution électronique Cairn.info pour ESKA.

(C) ESKA. Tous droits réservés pour tous pays.

La reproduction ou représentation de cet article, notamment par photocopie, n'est autorisée que dans les limites des conditions générales d'utilisation du site ou, le cas échéant, des conditions générales de la licence souscrite par votre établissement. Toute autre reproduction ou représentation, en tout ou partie, sous quelque forme et de quelque manière que ce soit, est interdite sauf accord préalable et écrit de l'éditeur, en dehors des cas prévus par la législation en vigueur en France. Il est précisé que son stockage dans une base de données est également interdit. 


\section{LES VARIABLES PSYCHOLOGIQUES MÉDIATISÉES PAR LE SERIOUS GAME DANS L'INSERTION ET LA FORMATION}
Mariama
Daniel
Lobsang
GILIBERT $T^{133}$
Jérôme
$N D A O^{132}$
DINET $T^{134}$

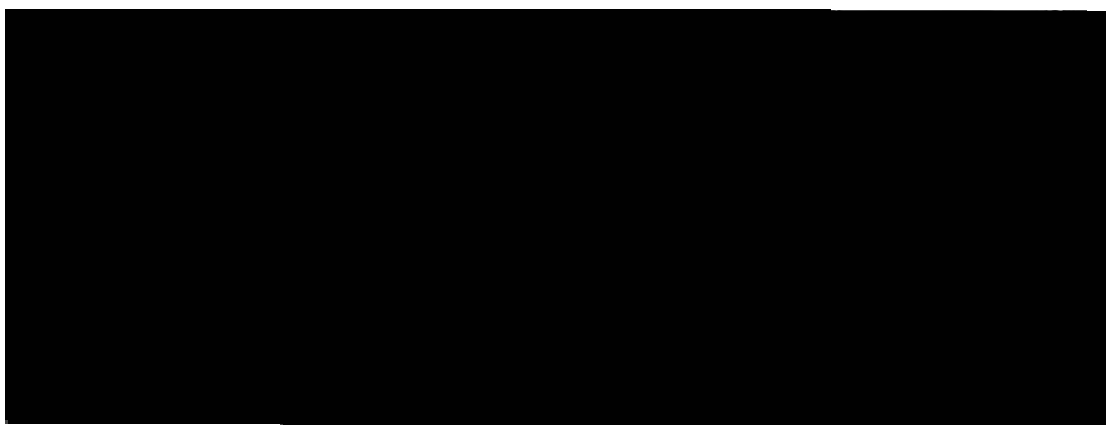

\section{INTRODUCTION}

S'il semble aujourd'hui établi que les serious games ont un impact positif sur les apprentissages et la motivation (Alvarez \& Djaouti, 2010; Marne et al., 2011,

\footnotetext{
${ }^{132}$ Psychologue du travail et directrice du centre de recherche CRAIES-NDAO, est doctorante et enseignante au laboratoire INTERPSY. Ses recherches portent sur les comportements au travail ou en situation de perte d'emploi. Plus précisément, ses travaux concernent les serious game et le monde de l'insertion et de la formation, la santé et le bien être, le marché de l'emploi et le management.

${ }^{133}$ Professeur en Psychologie sociale des Organisations et des Ressources Humaines, membre associé au Laboratoire INTERPSY (Université de Lorraine) et actuellement professeur à l'université de Montpellier 3, Laboratoire EPSYLON et responsable de la Mention en Psychologie Sociale et du Travail. Ses travaux de recherche portent sur l'application des recherches sur l'internalité et le contrôle aux relations de travail, d'accompagnement en formation et de soin.

${ }^{134}$ Professeur de psychologie à l'Université de Lorraine. Ses recherches concernent la modélisation des comportements et des processus cognitifs impliqués lors de l'interaction entre les apprenants et des environnements numériques (recherche d'information et navigation au sein des environnements numériques et impacts liés aux usages de ces systèmes).
} 
Galois-Faurie \& Lacroux ; 2014, Cohard, 2015), leur usage pour l'insertion des demandeurs d'emploi n'est qu'à ses débuts. De plus, même si de nombreux travaux expliquent que les serious games provoquent des changements internes chez l'apprenant et orientent son comportement (Marne et al., 2011 ; Becerril-Ortega \& Caron, 2011; Sanchez et al., 2011), l'analyse de l'influence de ces changements internes sur les dispositions psychologiques reste à préciser et le pouvoir de prédiction d'une réussite objective par ces variables psychologiques reste à démontrer ou à confirmer.

L'objectif de cet article est de proposer un état de l'art sur l'usage des serious games comme catalyseurs des facteurs psychologiques dans l'insertion et la formation, mais aussi d'analyser les impacts sur l'employabilité des demandeurs d'emploi. Afin de décrire le lien entre serious game et variable psychologique dans le domaine de l'emploi, il nous paraît nécessaire dans un premier temps de nous attarder sur les approches ludoéducatives afin d'en expliquer les enjeux en termes d'apprentissage, de motivation et d'impact psychologique. Dans une seconde partie, nous allons mettre en évidence l'essor des serious games dans le monde professionnel et l'intérêt de leur usage dans l'insertion socioprofessionnelle et/ ou la formation. Enfin, nous aborderons en conclusion les éventuels effets catalyseurs du serious game sur les facteurs psychologiques permettant de prédire un comportement actif vers l'emploi grâce un accompagnement «psycho-socio-professionnel ».

\section{LES APPROCHES LUDO-ÉDUCATIVES : LE SERIOUS GAME}

\section{La psychologie et les jeux}

Metra (2006) analyse différents points de vue sur la thématique du jeu et met en évidence ses aspects culturels, sociaux, philosophiques et psychologiques. En psychologie et notamment en psychologie du développement, la notion de jeu occupe une place centrale. En effet, pour des auteurs comme Piaget (1945), Winnicott et Monod (1975) ou Vygotsky (1978), le jeu a un impact sur le développement cognitif, conatif et affectif des individus. Il joue donc un rôle intéressant dans la formation des connaissances. Pour Winnicott et Monod (1975), le jeu constitue le lieu où l'environnement extérieur interagit avec le monde intérieur de l'enfant afin de lui donner les moyens d'apprendre et de se développer. Vygotsky (1978), quant à lui, parle de zone proximale de développement, qui va amener l'enfant à s'appuyer sur ses acquis pour augmenter ses connaissances avec l'aide d'un tiers, sans que celui-ci ne se substitue à lui. Ainsi, on constate que le jeu intervient positivement à l'intégration des premiers savoirs dès leur conception (y compris sur des dimensions sérieuses). 


\section{Les serious games}

Les personnes, mais aussi les entreprises, sont bouleversées par la croissance des nouvelles technologies et d'internet, qui offrent plusieurs possibilités (Barlatier, 2016). Aujourd'hui, de nombreux secteurs d'activités ont recours aux serious games (Galois-Faurie \& Lacroux, 2014) dans le champ de la formation par exemple (Sanchez et al., 2011). Alvarez (2007) décrit leur omniprésence au 21ème siècle non pas comme un effet de mode, mais plutôt comme un phénomène naturel faisant partie intégrante de notre culture. Le serious game est défini comme étant "une application informatique, dont l'intention initiale est de combiner, avec cohérence, à la fois des aspects sérieux (Serious) tels, de manière non exhaustive et non exclusive, l'enseignement, l'apprentissage, la communication, ou encore l'information, avec des ressorts ludiques issus du jeu vidéo (Game) » (Alvarez, 2007, p.263). Dans ce cas, l'apprentissage s'inscrit dans un scénario techniquement et méthodologiquement inspiré des jeux de rôles ou jeux vidéo (Alvarez \& Djaouti, 2010). McFarlane et al. (2002) précisent que les jeux éducatifs permettent de :

- $\quad$ Susciter un intérêt et favoriser la motivation à apprendre,

- Maintenir l'attention et la concentration des apprenants,

- $\quad$ Faciliter le travail en groupe et le partage des ressources communes.

Ajoutons que selon Alvarez (2007), il est nécessaire de disposer d'un scénario pédagogique lorsqu'on parle de serious game. Il s'agit en réalité d'un contenu pédagogique regroupant des objectifs clairement définis afin d'entraîner, d'instruire ou d'informer. Le serious game pourrait donc être un moyen d'accompagner les demandeurs d'emploi dans l'apprentissage des techniques de recherche d'emploi (TRE) à l'instar des formations classiques. Selon Marchais-Roubelat (2011), les choix pris virtuellement peuvent avoir une réelle influence sur le comportement et l'activité de l'individu en situation réelle et aussi donner lieu à un rapide transfert d'apprentissage. En outre, pour Martin et Lhuilier (2016), l'individu peut ajuster son comportement professionnel et utiliser facilement ses acquis grâce au serious game.

Toutefois, il est nécessaire de préciser que les serious games n'ont pas toujours été sur support numérique. En effet, historiquement les prémices du concept de serious games dateraient des XVème et XVIème siècles à l'époque de la renaissance italienne sous l'oxymore « serio ludere » (Alvarez, 2007, p.18). Ce dernier évoquait l'usage de l'humour pour traduire des dimensions sérieuses. D'ailleurs, Abt (1970) ne considère pas le serious game comme une simple application informatique, mais plutôt comme un concept plus large qui rend possible le fait d'apprendre sérieusement en jouant. Selon Mauco (2008), à la différence des jeux traditionnellement divertissants, le serious game propose un message sérieux. Par conséquent, il ne se réduit pas uniquement à une application informatique, mais suppose une dimension plus générale intégrant des mécanismes d'apprentissage ainsi que des facteurs motivationnels et dispositionnels. 


\section{LE LIEN SERIOUS GAME, APPRENTISSAGE, MOTIVATION ET VARIABLES PSYCHOLOGIQUES}

Depuis plusieurs décennies l'utilisation des jeux suscite beaucoup d'intérêt et fait l'objet de nombreux travaux de recherche (Cohard, 2015). Ces derniers ont étudié la nature des facteurs psychiques dans les jeux pour identifier le socle des mécanismes d'apprentissage impliqués et les facteurs motivationnels. Selon Sanchez et al. (2011) les jeux opèrent des changements d'états psychologiques chez les joueurs.

\section{Les apprentissages et la motivation dans le jeu}

Marne et al. (2011) affirment que l'efficacité d'un serious game repose sur l'introduction de notions de plaisir et de motivation dans l'interaction entre l'apprenant et le jeu. Les caractéristiques du serious game renforcent donc la motivation et favorisent l'apprentissage (Alvarez, 2007 ; Becerril-Ortega \& Caron, 2011, Cohard, 2015) grâce au concept de flow et au sentiment d'immersion qu'il provoque. Le concept de «flow» décrit un état de bonheur subjectif dans lequel l'individu se trouve particulièrement motivé, concentré, voire absorbé par une activité (Csikszentmihalyi, 1990). D'après Denis (2006), le «flow» suppose une motivation intrinsèque élevée où l'individu mobilise l'ensemble de ses facultés pour accomplir l'objet de sa motivation.

Concernant le sentiment d'immersion, Marne et al., (2011) admettent que le jeu induit ce sentiment qui est facteur de motivation et qui favorise l'apprentissage. Pour Witmer et Singer (1998), l'immersion est un état psychologique dans lequel l'individu se sent immergé dans un système stimulant qui augmente sa motivation et son engagement à accomplir les objectifs qu'il s'est fixé. Ainsi, les serious games peuvent influencer positivement les comportements et accentuer la motivation grâce aux concepts de flow et d'immersion. On pourrait donc supposer qu'ils constitueraient également un atout dans le cadre d'un dispositif d'insertion des demandeurs d'emploi. Cependant, dans leur méta-analyse sur les effets du serious game, Wouters et al. (2013, p.261) confirment les impacts du serious game sur les apprentissages, mais soulignent que ce dernier n'est pas plus motivant que les méthodes classiques. En effet, pour les auteurs plusieurs arguments peuvent expliquer le fait que le jeu n'augmente pas plus la motivation que les méthodes classiques.

- $\quad$ Premièrement, en se basant sur les travaux de Deci et al. (1999) et ceux de Ryan et al. (2006) sur la motivation, Wouters et al., (2013) montrent que le degré d'autonomie et de liberté d'action sont déterminants dans la motivation intrinsèque. Or, pour eux, le niveau de contrôle dans les jeux est double et applicable aux actions 
et décisions. De plus, à la différence des jeux de loisir qui ne présentent généralement pas de contrainte de temps, les serious games proposent souvent des temps de jeu limités.

- $\quad$ Le deuxième argument porte sur la conception des jeux dans lesquels, selon Wouters et al. (2013), la priorité est donnée soit à la structure pédagogique soit à l'environnement ludique.

Enfin, le dernier argument porte sur les méthodes utilisées pour évaluer la motivation. Pour Wouters et al. (2013), les mesures comportementales, comme les " eye tracking », seraient plus appropriées que des évaluations d'états affectifs par questionnaires souvent utilisées pour estimer la motivation dans les jeux.

Cette méta-analyse relativise donc l'effet du serious game sur la motivation.

\section{Les mécanismes d'apprentissage dans le jeu}

Pour Postle (1993), l'état émotionnel détermine la capacité d'apprentissage d'une personne. Ce registre émotionnel (immersion et flow) de l'apprenant provoqué par le serious game favorise le processus d'apprentissage permettant de changer sa structure cognitive pour intégrer de nouveaux savoirs. Pour les constructivistes comme Piaget et les socioconstructivistes comme Vygostsky, l'apprentissage est un processus actif et constructif dans lequel la stratégie «learning by doing » (apprendre en faisant) propose une vision où l'apprenant, bâtisseur de sa réalité, crée des liens cognitifs entre les savoirs en cours d'acquisition et ceux préalablement intégrés (Strommen \& Lincoln, 1992; Lombardo \& Dumas, 2006). Pour Juul (2005), le serious game améliore ce répertoire et développe les capacités d'apprentissage de l'apprenant (Alvarez \& Maffiolo, 2011 ; Becerril-Ortega \& Caron, 2011). De plus, dans une perspective cognitiviste de l'apprentissage, les tâches complexes sont divisées en sous-tâches qui peuvent prendre la forme de défis, amenant l'apprenant à se surpasser grâce au jeu et à parfaire ainsi son répertoire de connaissances (Juul, 2005).

\section{Les facteurs psychologiques dans les jeux}

Les serious game renforcent les dispositions individuelles par le biais de la motivation intrinsèque, du «flow », du sentiment d'immersion, des états émotionnels et psychologiques (Alvarez, 2007 ; Becerril-Ortega \& Caron, 2011 ; Marne et al., 2011 ; Sanchez et al., 2011). Le jeu mobilise ainsi des affects positifs, favorisant la motivation et le sentiment de compétence. A ce titre, Dindar et Akbulut (2015) montrent une corrélation élevée entre sentiment d'efficacité et motivation dans le cadre des jeux. Pour Galois-Faurie et Lacroux (2014), le stress peut être influencé positivement par le serious game. Bell et al. (2008) ajoutent qu'avec ce genre de technologie, les joueurs peuvent mieux contrôler leur apprentissage et donc mieux orienter leurs actes (Cohard, 2015). De plus, lorsque les objectifs sont clairement 
définis, le serious game permet une meilleure concentration sur la tâche à exécuter et un plus grand sentiment de contrôle de ses gestes (Cohard, 2015).

Par ailleurs, Soulage (2015) souligne que les gens pensent majoritairement que les personnes en situation de pauvreté ou de chômage ont une grande responsabilité dans cet état de faits. Ainsi, pour l'auteur, le fait d'être inactif peut conduire à une exclusion, surtout lorsque l'individu dispose d'un faible réseau. Ce qui contribue au mal-être des demandeurs d'emploi. Mcfarlane et al. (2002), mettent en avant l'effet particulièrement négatif du stress sur le déploiement des capacités cognitives. Pour ces mêmes auteurs, l'environnement ludique qu'offre le jeu permet de désamorcer des situations difficiles telles que le chômage. De plus, dans le cadre d'un reclassement, certains demandeurs d'emploi peuvent contester la démarche de recherche, la jugeant dépréciative, dénigrante et certaines propositions d'emploi inconcevables (Crunel \& Frau, 2014). Le jeu peut intervenir à ce niveau car il permet d'identifier les difficultés et de les résoudre sans tension ni pression, de tester et d'intégrer les connaissances et compétences et d'en observer les conséquences dans des conditions favorables. Pour l'auteur, la pédagogie par le jeu met en confiance les apprenants car l'erreur y est permise et les efforts encouragés, elle reste pour autant une pédagogie sérieuse qui suppose concentration et attention dans le respect des règles établies. L'environnement ludique est donc propice à l'apprentissage des techniques de recherche d'emploi (TRE) et permet de lever les freins liés au stress, ou plus généralement aux facteurs psychologiques négatifs pouvant entraver l'accès à l'emploi.

Nous constatons grâce à ces travaux un lien indéniable entre serious game, motivation, apprentissage et variables dispositionnelles. Il convient donc de mener une réflexion sur ce lien dans l'accompagnement des demandeurs d'emploi et leur insertion.

\section{L'INSERTION ET LA FORMATION : LA GAMIFICATION D'UN ACCOMPAGNEMENT « PSYCHO-PROFESSIONNEL »}

\section{Les facteurs psychologiques dans l'insertion et la formation}

Beaucoup de travaux de recherche ont porté sur les facteurs dispositionnels, la performance et le devenir social. Certains d'entre eux discutent de l'importance des variables psychologiques dans la réussite académique, dans l'insertion ou la réinsertion professionnelle (Gillet, 2011). A ce titre, dans leur méta-analyse, Kanfer et al. (2001) ont montré des corrélations positives entre l'estime de soi, le sentiment d'efficacité personnelle, le locus de contrôle et les résultats de recherche d'emploi. De plus, pour ces auteurs, la manière de rechercher un emploi est significativement 
et positivement corrélée au fait de trouver un emploi. Notons que certaines variables dispositionnelles, comme le sentiment d'efficacité personnelle, peuvent mettre en lumière la valeur d'un contenu pédagogique dans le cadre d'une évaluation de formation (Kraiger et al., 199 ;, Beech \& Leather, 2006).

Par ailleurs, de nombreux travaux de recherche axés sur la perte d'activité montrent des conséquences néfastes de cette dernière sur l'individu (dépression, souffrance, suicide, santé mentale dégradée) (McKee-Ryan et al., 2005 ; Paul \& Moser, 2009). En effet, selon Roques (2008), lors de la perte d'un emploi, sont impactés négativement le bien-être psychologique et la confiance en soi (Wanberg et al., 1996). Pour Murphy et Athanasou (1999), le retour à l'emploi influence positivement la santé mentale. Dans ce cadre, même si l'accompagnement vers l'emploi n'a pas une visée thérapeutique, il convient de souligner qu'il a un impact sur l'image de soi, la motivation et le sentiment de compétence (Guénolé et al. 2012). Creed et al. (1998) ont montré quant à eux l'évolution positive des facteurs dispositionnels lors d'un accompagnement, même si cet effet est modéré sur le long terme.

La perception de soi revêt donc une importance capitale dans les domaines de la formation et de l'insertion. C'est pour cette raison qu'il semble incontournable, dans cette revue de la littérature, d'évoquer les variables psychologiques qui y sont rattachées. Notre objectif n'est pas ici de dénombrer l'ensemble de ces variables, mais de rappeler notamment celles qui ont fait l'objet de nombreux travaux de recherche, comme l'évaluation centrale de soi, le sentiment d'efficacité personnelle, le locus de contrôle et l'estime de soi.

\section{L'évaluation centrale de soi}

Judge et al. (1997) introduisent le concept d'évaluation centrale de soi qui regroupe l'estime de soi, le sentiment d'efficacité personnelle, le névrosisme et le locus de contrôle. Selon ces auteurs, l'évaluation centrale de soi correspond à un trait global de personnalité stable dans le temps nomé "core self-evaluation "; il renvoie à l'appréciation qu'une personne se fait d'elle-même, d'autrui, de ses interactions, de ses capacités de contrôle, de ses compétences et de son milieu. Notons que l'estime de soi, le contrôle interne, le sentiment d'efficacité personnelle et l'évaluation centrale de soi peuvent rendre proactif un demandeur d'emploi dans le cadre de son insertion professionnelle (Meyers \& Houssemand, 2005). De plus, pour ces auteurs (2010), ces variables permettent également de prédire le chômage de longue durée. Pour Liu et al. (2016), le soutien social que l'on peut retrouver dans le cadre d'un accompagnement a un impact positif sur l'évaluation centrale de soi, qui a son tour influence favorablement le bien-être. 


\section{Le sentiment d'efficacité personnelle}

Issue de la théorie socio-cognitive de Bandura (1991, 1999), le sentiment d'efficacité personnel (SEP), ou sentiment de compétence, renvoie aux croyances qu'une personne entretient quant à ses capacités à organiser et à exécuter des actions futures en vue d'un résultat positif. Dans le domaine de la formation, le sentiment d'efficacité se traduirait par " la croyance des individus en leurs propres capacités à produire un résultat ou une performance, à savoir par exemple de retenir les notions transmises par une formation, réussir une formation ou encore réussir à un examen » (Gillet, 2011, p. 57). Pour Eden et Aviram (1993), après une formation, l'attitude face à la recherche d'emploi, ainsi que la possibilité d'accéder à un emploi, pourront être modulées par le SEP. En effet, leurs expérimentations sur cette variable ont montré que cette dernière s'accroît durant la formation, qu'elle influence les comportements de recherche d'emploi et donc les chances d'être inséré. Dans le même ordre d'idée, Crossley et Stanton (2005) ajoutent que le SEP peut orienter l'efficience des techniques de recherche d'emploi (TRE). Selon Desmette et al. (2001) un demandeur d'emploi s'engage plus facilement dans une dynamique d'insertion s'il a un fort sentiment d'efficacité personnelle. En fin de formation, ce sentiment favorise l'engagement dans un processus d'insertion socioprofessionnelle alors que le sentiment d'inefficacité, quant à lui, peut être un frein à l'emploi (Desmette et al., 2001). De plus, les individus ayant un fort sentiment d'efficacité ont un comportement plus actif dans leurs recherches (Wanberg et al., 2000), et sont donc susceptibles de sortir plus rapidement du chômage. En d'autres termes, cette variable a une influence positive sur l'insertion (Vinokur et al., 1991). Pour finir, Bandura (1999) précise que, corrélé avec d'autres facteurs (personnalité, disposition, environnement), le SEP peut directement et indirectement orienter les comportements de l'individu et par conséquent agir sur :

- $\quad$ ses préférences, ses ambitions,

- $\quad$ ses comportements entrepris pour atteindre un but,

- $\quad$ sa volonté à réussir une action ou à dépasser un obstacle,

- $\quad$ sa performance.

Le sentiment d'efficacité personnelle impacte donc sur les conduites humaines et la performance par l'intermédiaire de facteurs cognitifs, motivationnels, affectifs et sélectifs (Bandura, 1991). Cette variable prédit effectivement de nombreux comportements lorsque la personne agit en fonction de ses croyances en ses capacités par rapport à une situation donnée (Guénolé et al., 2012).

\section{L'estime de soi}

L'estime de soi est une évaluation de soi (auto-évaluation positive ou négative) ou une estimation de sa valeur dans tout contexte social (Rosenberg, 1965). Elle reflète la connaissance subjective de soi et peut être liée à la réussite sociale et à la performance (Gillet, 2011). L'estime de soi permet donc d'évaluer ses qualités, son 
potentiel, ses compétences. Pour Guénolé et al. (2012), la littérature sur la relation entre estime de soi et situation de perte d'emploi est dense. Selon eux, l'accompagnement vers l'emploi a une influence sur l'estime de soi. Ellis et Taylor (1983) affirment que cette variable a également un impact sur les moyens mis en place pour trouver un travail, le nombre d'offres reçues, ainsi que l'efficacité de la recherche d'emploi. Creed et al. (1998) soulignent aussi le lien entre estime de soi et comportement de recherche d'emploi. Pour Roques (1995), une estime de soi élevée favorise davantage l'accès à l'emploi car elle est préalable à un comportement stratégique et permet l'autonomie dans ses techniques de recherche d'emploi (TRE). Wanberg (1997) ajoute que la situation de réinsertion professionnelle est plus facile pour les demandeurs d'emploi ayant une grande estime d'eux-mêmes.

\section{Le locus de contrôle}

Pour Gillet (2011) le locus de contrôle permet, selon les capacités d'une personne, de prédire :

- l'atteinte d'un objectif précis grâce à sa personnalité et à son environnement,

- un comportement susceptible de se manifester, comme l'accès à une formation ou un emploi.

Plus précisément, il s'agit de la croyance qu'un individu a en ses capacités à contrôler des évènements positifs ou négatifs de sa vie (Rotter, 1966). Il peut être interne, dans ce cas l'individu contrôle ses comportements et leurs conséquences, ou externe, dans ce cas les comportements et leurs conséquences dépendent de facteurs extérieurs à l'individu (Rotter, 1990). Un locus de contrôle interne favoriserait le transfert de connaissances et de compétences en situation réelle (Gillet, 2011). Il peut également influencer l'insertion professionnelle (Millet \& Sandberg, 2003) et prédire les impacts d'une formation destinée à la réinsertion (emploi ou poursuite d'étude). Pour Millet et Sandberg (2003), les personnes ayant un locus de contrôle interne sont celles qui s'adaptent le plus facilement à la réinsertion. En effet, pour les auteurs, les personnes internes espèrent la réussite et enclenchent davantage des processus cognitifs et conatifs pour l'atteindre que les personnes externes. Dans leur étude, Millet et Sandberg montrent ainsi que les personnes internes ont plus de facilité à créer des "espaces d'action » qui sont en eux-mêmes des opportunités pouvant être utilisées pour la réalisation d'objectifs que les externes. Selon Colquitt et al. (2000), dans le domaine de la formation, le locus de contrôle augure d'une grande motivation à apprendre et agit directement sur les apprentissages.

Nous pouvons donc constater qu'il existe un lien entre variables dispositionnelles et accompagnement des demandeurs d'emploi. Cependant Gillet (2011) montre dans sa thèse que ce lien, même s'il existe, est faible et la causalité difficile à déterminer. Il faut donc nuancer le pouvoir prédictif de ces variables quant à la réussite objective ou le comportement (Channouf et al., 1996 ; Gilibert, 2009 ; Gillet, 2011). En effet, 
les personnes évaluées dans des conditions de réussite ont tendance à partager des croyances socialement valorisées et personnellement gratifiantes (Gilibert, 2009; Gillet et al., 2011). Ainsi, les explications de type interne que l'on retrouve dans les variables dispositionnelles comme le locus de contrôle, l'évaluation centrale de soi, le sentiment d'efficacité personnelle et l'estime de soi renforcent "l'idée que ces variables font l'objet d'une norme et qu'elles sont valorisées en contexte éducatif, indépendamment de la performance " (Gillet, 2011, p84). Pris isolement ces variables ne peuvent pas prédire à elles seules des résultats et des comportements (Gilibert, 2009 ; Gillet et al., 2011). Finalement, il serait intéressant de travailler sur ces variables combinées avec d'autres facteurs comme les serious games pour rehausser leur prédictibilité de la réussite objective ou de l'insertion des demandeurs d'emploi.

\section{Les variables psychologiques et la notion d'employabilité}

Nous allons nous attarder sur le concept d'employabilité, incontournable dans une revue de la littérature portant sur l'insertion. En effet, dans le cadre des accompagnements vers l'emploi, le demandeur d'emploi est positionné dans une dynamique d'adaptabilité et serait personnellement responsable ou acteur de son insertion (Salognon, 2006). Ainsi, l'adaptabilité des caractéristiques individuelles du chercheur d'emploi aux besoins du marché déterminera son degré d'employabilité. Fugate et al. (2004) précisent qu'un demandeur d'emploi avec un degré d'employabilité élevé aura une meilleure préparation psychologique face à sa recherche d'emploi, sera plus motivé dans sa démarche et s'insèrera plus facilement (McArdle et al., 2007).

\section{Vers une gamification des dispositifs d'insertion et de formation}

Pour lutter contre le chômage, les pouvoirs publics misent sur l'augmentation du nombre de formation afin de rehausser le niveau de compétences des demandeurs d'emploi (Gillet, 2011) et sur la mise en place des dispositifs d'insertion pour favoriser le retour à l'emploi des demandeurs d'emploi (Guénolé et al., 2012). Les politiques de l'emploi agissent donc dans le but d'améliorer les méthodes d'accompagnement des demandeurs d'emploi (Ferracci \& Martin, 2013). La recherche d'innovation dans ce secteur constitue alors un enjeu politique, social et économique. Malgré cela, à notre connaissance, peu d'études se sont centrées sur l'apport des "serious games» en matière d'accompagnement et de suivi individualisé des demandeurs d'emplois. Pourtant, la présence des serious games modifie considérablement l'univers de la formation, en offrant de nouvelles possibilités quant aux façons d'innover en matière d'enseignement (Alvarez \& Djaouti, 2010) et en soulevant des enjeux importants dans le domaine de l'insertion professionnelle (Cohard, 2015). Dans le monde de l'entreprise, certains processus s'améliorent grâce aux jeux, par le biais de la ludification des systèmes (Simões et 
al., 2013 ; Galois-Faurie \& Lacroux, 2014). Ainsi, la gamification, qui suppose une interaction homme-machine favorisant motivation, hédonisme et épanouissement (Marache-Francisco \& Brangier, 2015), pourrait être un atout dans les nouvelles formes d'accompagnement vers l'emploi. Selon Natkin (2006), le serious game permet la transmission de bonnes pratiques. Dans leur étude sur "l'usage d'un serious gaming dans un programme de formation au management» Martin et Lhuilier (2016) montrent un lien entre "le scénario du jeu et le travail réel des managers » (p.143). Ainsi, la gamification des dispositifs d'accompagnement vers l'emploi est plus que jamais pertinente. Pour Zyda (2005) le serious game offre une valeur ajoutée à une formation grâce au divertissement et à la stimulation cognitive qu'il provoque. Dans le domaine du recrutement, le serious game présente un apport stratégique par l'innovation qu'il propose, opérationnel par la mise en place de procédures efficaces et financier par la réduction des coûts (Galois-Faurie \& Lacroux, 2014). Il apparaît donc nécessaire de développer de nouveaux contenus pédagogiques en phase avec les nouvelles technologies tels que les serious games afin d'assurer un processus d'insertion socioprofessionnelle qui soit efficace et pertinent.

\section{L'accompagnement «psycho-socioprofessionnel »}

L'accompagnement vers l'emploi a un impact positif sur les demandeurs d'emploi (Guénolé et al., 2012 ; Parent \& Sautory, 2015) et peut influencer le taux de sortie vers l'emploi (Blasco et al., 2015). Havet (2014) va dans le même sens en soulignant que dans le cadre des dispositifs VAE, l'accompagnement favorise la réussite de ces derniers et augmente les possibilités d'une validation totale. En effet, au delà de l'accès à l'emploi, les prestations d'accompagnement ont une influence sur la perception de soi, les facteurs psychologiques et sociaux et servent d'appui, voire de repère pour les demandeurs d'emploi (Gaudron et al., 2001). Ainsi, la connaissance des caractéristiques professionnelles et psychologiques du demandeur d'emploi est importante dans une démarche d'accompagnement (Multon et al., 2007). De plus, la souffrance psychologique induite par une situation de chômage peut être réduite par l'accompagnement vers l'emploi (Paul \& Moser, 2009). Pour Roques (2008), l'allongement de la durée du chômage est une menace pour le bien-être psychologique des demandeurs d'emploi, ce qui aura possiblement un impact sur les comportements de recherche d'emploi. Ainsi, selon l'auteur, la prise en compte des facteurs psychologiques devrait constituer un objectif de formation. Pour Pochic et Bory (2014), la souffrance induite par l'inactivité qui culpabilise et isole est souvent négligée par rapport à la souffrance au travail. En somme, la prise en compte simultanée des facteurs sociaux, psychologiques et professionnels est essentielle dans une démarche de recherche d'emploi et l'introduction d'une notion «psychosocioprofessionnelle » mérite donc réflexion. 


\section{CONCLUSION}

Nous avons proposé un état de l'art sur les serious games, leur lien avec les facteurs dispositionnels, leur impact sur les apprentissages et la motivation, leur efficacité dans le domaine de l'insertion et la formation et enfin, leur place dans un accompagnement «psycho-socioprofessionnel ».

Les approches ludoéducatives telles que les serious games, ou jeux sérieux en français, ont un impact considérable sur les apprentissages et renforcent notablement la motivation des apprenants (McFarlane et al., 2002 ; Natkin, 2006 ; Alvarez \& Djaouti, 2010; Alvarez \& Maffiolo, 2011 ; Becerril-Ortega \& Caron, 2011) et ce même s'il faut nuancer leur influence sur la motivation selon certaines études (Wouters et al., 2013). De plus, elles opèrent également des changements d'états psychologiques chez les personnes (Sanchez et al., 2011). Ces états psychologiques ou variables dispositionnelles jouent un rôle important dans l'insertion professionnelle (Kanfer et al., 2001 ; Fleuret \& Zamora, 2005 ; Roques, 2008 ; Gillibert, 2009 ; Meyers \& Houssemand, 2010 ; Gillet, 2011) et leur prise en compte dans l'accompagnement, au même titre que les facteurs sociaux et professionnels, est nécessaire (Gaudron et al., 2001 ; Multon et al., 2007 ; Roques, 2008). Ainsi, les différentes problématiques abordées par la plupart des auteurs attestent donc d'un lien entre serious game, variables dispositionnelles et insertion, mais l'impact sur la réussite objective reste à confirmer. En effet, les variables dispositionnelles ont certes une influence sur la recherche d'emploi, mais leur pouvoir de prédiction d'une insertion n'est pas systématique et est souvent nuancée sur le long terme. Il serait donc judicieux d'effectuer une analyse plus fine sur les effets catalyseurs du serious game tant sur les variables dispositionnelles, les apprentissages, la motivation que sur l'insertion effective. Enfin, il est vraisemblable que ce genre de dispositif (serious games) favorise la conformité des variables dispositionnelles, mais aussi qu'il conditionne la réussite d'une telle conformité.

Par ailleurs, même si les travaux cités ci-dessus soulignent la triangulation de facteurs essentiels dans la recherche d'emploi que sont le social, le professionnel et le psychologique, l'introduction d'une notion "psycho-socioprofessionnelle » dans l'accompagnement semble s'imposer mais reste à définir. Il serait pertinent d'étudier ce concept pour développer des stratégies efficaces d'accompagnement vers l'emploi.

Pour conclure, plusieurs perspectives de recherche pourraient découler de notre revue de la littérature, afin de cerner le sens des interactions entre les différents concepts étudiés.

La première perspective est l'augmentation du pouvoir de prédiction des variables psychologiques de la réussite objective et ce, par le biais du serious game. Force est 
de constater que les effets des facteurs dispositionnels, surtout sur le long terme, sont très controversés. Or nous avons montré ici l'importance des dimensions psychologiques dans la recherche d'emploi et la nécessité de leur prise en compte pour un accompagnement vers l'emploi réussi. Les serious games ayant un impact positif plus que vraisemblable sur les apprentissages, la motivation et le comportement, leur usage comme catalyseur des variables psychologiques mérite réflexion. A ce titre, il serait intéressant de s'inspirer de l'ensemble des travaux cités ci-dessus, qui démontrent l'importance du caractère immersif des serious games et de leur impact sur la conscience et les comportements. Le jeu peut donc influer l'attitude du demandeur d'emploi et induire chez lui un comportement actif vers l'insertion. Dans ses études, Gillet (2011) a pu démontrer qu'en augmentant le temps consacré aux exercices en lien avec l'examen final, les variables dispositionnelles deviennent prédictives de la réussite ultérieure des apprenants. De plus, pour l'auteur, de simples feed-back évaluatifs pourraient favoriser le développement des variables dispositionnelles, qui prédiront alors des performances ultérieures. Ces feedback sont régulièrement présents dans les serious games, qui pourront par conséquent avoir également un effet d'accroissement positif des variables psychologiques sur l'insertion des demandeurs d'emploi. En résumé, les serious games pourront influencer les variables dispositionnelles qui peuvent à leur tour, prédire le devenir social en rendant compte puis en agissant sur la performance ou la réussite.

\section{Schéma explicatif}

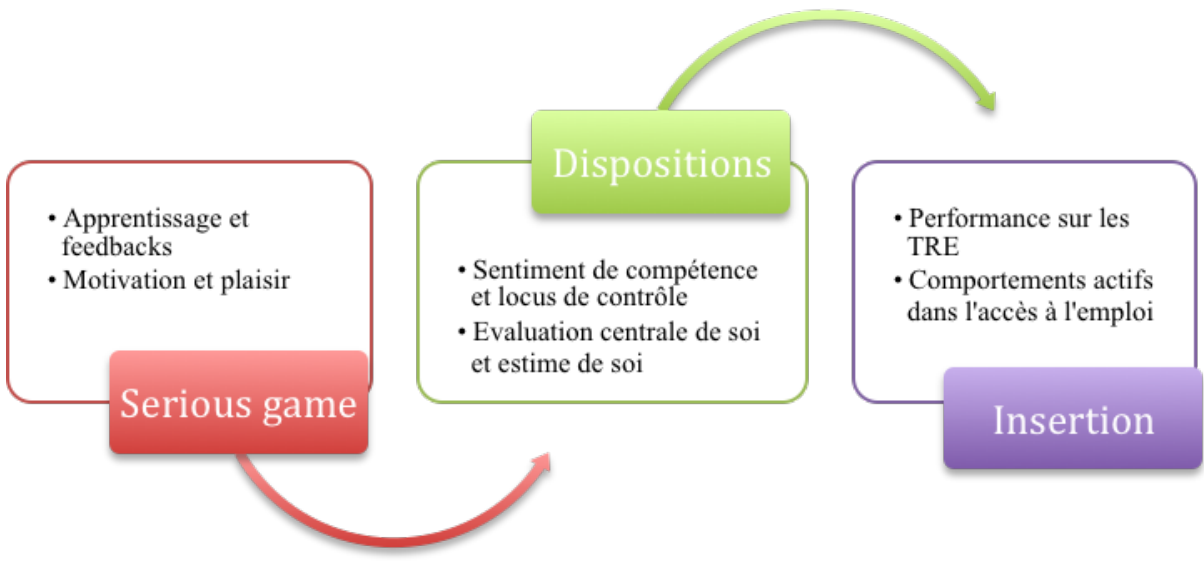

La deuxième perspective concerne l'introduction d'une dimension "psychosocioprofessionnelle» dans l'accompagnement. Si quelques études s'attachent à 
décrire les aspects socioprofessionnels voir psychoprofessionnels, peu d'entre elles introduisent une vraie notion "psycho-socioprofessionnelle » dans l'insertion. En effet, le modèle de Allard et Ouellette (2002) étudie les facteurs psychosociologiques du milieu et ceux psycho-professionnels de la personne. Ils présentent leur rôle dans la recherche d'emploi mais offrent une vision segmentée. Or, on ne peut plus faire l'économie de ces trois facteurs dans l'accompagnement des demandeurs d'emploi, surtout en période de crise économique. Si on prend en exemple l'étude sur l'effet des accompagnements dans le cadre du Plan d'Aide au Retour à l'Emploi (PARE) de Fougère, Kamionka, et Prieto (2010), on constate un effet positif de l'accompagnement sur le taux d'insertion, qui, selon Jaminon et Van Ypersele (2001), peut être influencé par la nature des interventions proposées lors de l'accompagnement. Notons que d'après Jugnot et al. (2006), les demandeurs d'emploi ont une vision positive de l'utilité des accompagnements vers l'emploi), même si les auteurs nuancent leur propos sur les effets directs de l'accompagnement sur l'insertion. Il faut donc prendre en compte ces trois facteurs essentiels dans la recherche d'emploi que sont le social (soutien social, réseau, environnement, etc.), le professionnel (techniques et stratégie de recherche, connaissance du marché, ...) et le psychologique (image de soi, confiance en soi, bien-être, ...) pour garantir un accompagnement complet et adapté. Il n'est donc plus question de parler d'un accompagnement uniquement socioprofessionnel ou psychoprofessionnel mais plutôt d'un accompagnement "psycho-socioprofessionnel » afin de mieux orienter les comportements du demandeur d'emploi.

A partir de l'ensemble de ces travaux, nous pouvons émettre les constats suivants :

- L'entraînement souvent présent dans les serious games peut augmenter le pouvoir prédictif des variables dispositionnelles sur la réussite objective.

- $\quad$ Le serious game permet l'entraînement et l'exercice, il a un impact sur les apprentissages et le développement des compétences.

- Le sentiment d'immersion présent dans les serious games, stimule l'apprentissage et permet l'atteinte des objectifs fixés.

- Le concept de flow décrit dans les serious games induit une haute motivation qui oriente positivement le comportement de l'apprenant. Cette motivation, favorisée par le serious game, augmente l'effet des variables dispositionnelles.

- L'environnement ludique engendre motivation et plaisir deux facteurs propices aux comportements engagés.

De ces constats découlent plusieurs questions de fond qui nous amènent à la problématique suivante :

l'utilisation des serious games comme catalyseur des facteurs psychologiques afin de favoriser l'insertion des demandeurs d'emploi est-elle 
envisageable, notamment dans un accompagnement «psycho-socioprofessionnel »? Plus précisément, peut-on parler d'un pouvoir amplificateur ou médiateur des serious games sur les facteurs dispositionnels afin de prédire une réussite objective ou le devenir social des demandeurs d'emploi ?

\section{BIBLIOGRAPHIE}

Abt, C. C. (1970). Serious games: The art and science of games that simulate life. USA, Viking Compass Book.

Allard, R., \& Ouellette, J. G. (2002). Vers un modèle macroscopique des facteurs déterminants de l'insertion socioprofessionnelle des jeunes. Carriérologie, $8,497-517$.

Alvarez, J. (2007). Du jeu vidéo au serious game: approches culturelle, pragmatique et formelle (Doctoral dissertation, Toulouse 2).

Alvarez, J., \& Djaouti, D. (2010). Introduction au serious game. Questions théoriques.

Alvarez, J., \& Maffiolo, V. (2011). Etude de l'impact de communications électroniques basées sur le Serious game. Revue de l'Electricité et de l'Electronique, 4.

Bandura, A. (1991). Social cognitive theory of self-regulation. Organizational behavior and human decision processes, 50(2), 248-287.

Bandura, A. (1999). Social cognitive theory: An agentic perspective. Asian journal of Social Psychology, 2(1), 21-41.

Barlatier, P. J. (2016). Management de l'innovation et nouvelle ère numérique. Revue française de gestion, (1), 55-63.

Becerril-Ortega Raquel, Caron Pierre-André, (2011), Dispositifs de formation et Serious Game, Analyse des porosités, Les jeux sérieux : situations, environnements ou artefacts pour l'apprentissage ?, 79e congrès de l'ACFAS - Sherbrooke, QC Canada.

Beech, B., \& Leather, P. (2006). Workplace violence in the health care sector: A review of staff training and integration of training evaluation models. Aggression and violent behavior, 11(1), 27-43.

Bell, B. S., Kanar, A. M., \& Kozlowski, S. W. (2008). Current issues and future directions in simulation-based training in North America. The International Journal of Human Resource Management, 19(8), 1416-1434.

Blasco, S., Fontaine, F., \& Malherbet, F. (2015). Améliorer l'efficacité du service public pour l'emploi. Revue française d'économie, 30(1), 67-97.

Channouf, A., Py, J., \& Somat, A. (1996). Prédire des comportements à partir des attitudes: nouvelles perspectives. La psychologie sociale, 2, 55-65.

Cohard, P. (2015). L'apprentissage dans les serious games: proposition d'une typologie.@GRH, (3), 11-40.

Colquitt, J. A., LePine, J. A., \& Noe, R. A. (2000). Toward an integrative theory of 
training motivation: a meta-analytic path analysis of 20 years of research. Journal of Applied Psychology, 85(5), 678.

Creed, P. A., Hicks, R. E., \& Machin, M. A. (1998). Behavioural plasticity and mental health outcomes for long-term unemployed attending occupational training programmes. Journal of Occupational and Organizational Psychology, 71(2), 171-191.

Crossley, C. D., \& Stanton, J. M. (2005). Negative affect and job search: Further examination of the reverse causation hypothesis. Journal of Vocational Behavior, 66(3), 549-560.

Crunel, B., \& Frau, C. (2014). Devenir chômeur: des parcours pluriels entre transition et état. Sociologies pratiques, (28), 75-85.

Csikszentmihalyi, M. (1990). Flow. The Psychology of Optimal Experience. New York (HarperPerennial) 1990.

Deci, E. L., Koestner, R., \& Ryan, R. M. (1999). A meta-analytic review of experiments examining the effects of extrinsic rewards on intrinsic motivation. Psychological Bulletin, 125(6), 627.

Denis, G. (2006). Jeux vidéo éducatifs et motivation: application à l'enseignement du jazz (Doctoral dissertation, Paris, ENMP).

Desmette, D., Jaminon, C. \& Herman, G. (2001). Le sentiment d'efficacité personnelle des chômeurs, un construct dynamique ? Revue Européenne de Psychologie Appliquée, 51(3), 217-228.

Dindar, M., \& Akbulut, Y. (2015). Role of self-efficacy and social appearance anxiety on gaming motivations of MMOFPS players. Computers \& Education, 81, 26-34.

Eden, D., \& Aviram, A. (1993). Self-efficacy training to speed reemployment: Helping people to help themselves. Journal of Applied Psychology, 78(3), 352.

Ellis, R.A., \& Taylor, M.S. (1983). Role of self-esteem within the job search process. Journal of Applied Psychology, 68(4), 632-640.

Ferracci, M., \& Martin, F. (2013). Évaluation par expérimentation aléatoire de dispositifs de reclassement innovants pour les demandeurs d'emploi. Travail et emploi, (3), 15-26.

Fleuret, A., \& Zamora, P. (2005). La formation professionnelle des chômeurs. France, portrait social 2004/2005, 151-172.

Fougère, D., Kamionka, T., \& Prieto, A. (2010). L'efficacité des mesures d'accompagnement sur le retour à l'emploi. Revue économique, 61(3), 599612.

Fugate, M., Kinicki, A. J., \& Ashforth, B. E. (2004). Employability: A psycho-social construct, its dimensions, and applications. Journal of Vocational behavior, 65(1), 14-38.

Galois-Faurie, I., \& Lacroux, A. (2014). «Serious games» et recrutement: quels enjeux de recherche en gestion des ressources humaines?. @ GRH, (1), 1135 . 
Gaudron, J. P., Bernaud, J. L., \& Lemoine, C. (2001). Évaluer une pratique d'orientation professionnelle pour adultes: les effets individuels du bilan de compétences. L'orientation scolaire et professionnelle, (30/4).

Gilibert, D. (2009). La norme d'internalité et "l'individu responsable, utile et heureux de l'être ", Application des recherches sur l'internalité et le contrôle aux relations de travail, d'accompagnement en formation et de soin. Habilitation à Diriger des Recherches, Université de Bourgogne, Dijon.

Gillet, I. (2011, December 08). L'impact des variables dispositionnelles et de la déclaration de liberté sur les résultats d'une activité de formation $(\mathrm{PhD}$ Thesis). Dijon, France : Université de Bourgogne.

Gillet, I., Gilibert, D., \& Banovic, I. (2011). Les effets de « l'évaluation centrale de soi » manifestée en situation de recrutement : une utilisation des paradigmes classiques d'études de is íp.]la norme d'internalité. $53^{\text {ème }}$ édition du Congrès National de la Société Française de Psychologie, Université Paul Verlaine, Metz, 6 au 9 septembre 2011.

Guénolé, N., Bernaud, J. L., \& Guilbert, L. (2012). L'accompagnement à la recherche d'un emploi: enjeux, modèles, et perspectives de recherche. Psychologie du Travail et des Organisations, 18(3), 193-214.

Havet, N. (2014). Le rôle de l'accompagnement dans la réussite des parcours de validation des acquis de l'expérience. Formation emploi, (1), 47-68.

Jaminon, C., \& Van Ypersele, D. (2001). Parcours d'insertion et trajectoires individuelles. L'insertion: Défi pour l'analyse, enjeu pour l'action, 219240.

Judge, T.A., Locke, E.A., Durham, C.C. (1997). The dispositional causes of job satisfaction: a core evaluations approach. Research in Organizational Behavior, 19, 151-188.

Jugnot, S., Renard, E., \& Traversier, T. (2006). L'accompagnement personnalisé des demandeurs d'emploi: de l'accès aux prestations aux sorties du chômage vers l'emploi. Données sociales-la société française, 323-331.

Juul, J. (2005). Half-real. Video Games between Real Rules and Fictional Worlds. Cambridge (Massachusetts) and London. OLDENBURG.

Kanfer, R.,Wanberg, C.R., \& Kantrowitz, T.M., (2001). Job search and employment: A personality-motivational analysis and meta-analytic review. Journal of Applied Psychology, 86(5), 837-855.

Kraiger, K., Ford, J. K., \& Salas, E. (1993). Application of cognitive, skill-based, and affective theories of learning outcomes to new methods of training evaluation. Journal of Applied Psychology, 78(2), 311.

Liu, W., Li, Z., Ling, Y., \& Cai, T. (2016). Core self-evaluations and coping styles as mediators between social support and well-being. Personality and Individual Differences, 88, 35-39.

Lombardo, E., \& Dumas, P. (2006, May). La métacognition dans l'évaluation de la formation a distance. In TiceMed 06 (No. 25, pp. 1-13). 
Marache-Francisco, C., \& Brangier, E. (2015). Gamification and human-machine interaction: a synthesis. Le travail humain, 78(2), 165-189.

Marchais-Roubelat, A., \& Roubelat, F. (2011). The Delphi method as a ritual: Inquiring the Delphic Oracle. Technological Forecasting and Social Change, 78(9), 1491-1499.

Marne, B., Huynh-Kim-Bang, B., \& Labat, J. M. (2011). Articuler motivation et apprentissage grâce aux facettes du jeu sérieux. In Environnements Informatiques pour l'Apprentissage Humain, Conférence EIAH'2011 (pp. 69-80). Editions de l'UMONS, Mons 2011.Martin, L., \& Lhuilier, D. (2016). Entraînement à la prise de décision avec un serious gaming: délibération et conflits de valeurs. Psychologie $d u$ Travail et des Organisations, 22(2), 135-146.

Mauco, O. (2008). Les serious games, entreprise d'auto-légitimation. Les jeux vidéo, un « bien » culturel ? Média- Morphoses, 22, 79-84.

McArdle, S., Waters, L., Briscoe, J. P., \& Hall, D. T. T. (2007). Employability during unemployment: Adaptability, career identity and human and social capital. Journal of Vocational Behavior, 71(2), 247-264.

McFarlane, A., Sparrowhawk, A., \& Heald, Y. (2002). Report on the educational use of games. TEEM (Teachers evaluating educational multimedia), Cambridge.

McKee-Ryan, F., Song, Z., Wanberg, C. R., \& Kinicki, A. J. (2005). Psychological and physical well-being during unemployment: a meta-analytic study. Journal of Applied Psychology, 90(1), 53.

Métra, M. (2006). Le jeu dans le développement affectif, cognitif, corporel et social de l'enfant. Lyon: UFAIS.

Meyers, R., \& Houssemand, C. (2005). Etude de la dimension psychologique sur l'insertion professionnelle et ses interactions avec les sphères sociales et économiques. Working Paper $n^{\circ} 3,1-76$.

Meyers, R., \& Houssemand, C. (2010). Socioprofessional and psychological variables that predict job finding. Revue Européenne de Psychologie Appliquée/European Review of Applied Psychology, 60(3), 201-219.

Millet, P., \& Sandberg, K. W. (2003). Locus of control and its relationship with vocational rehabilitation of unemployed sick leaves in Sweden. Journal of Vocational Rehabilitation, 19(1), 59-66.

Multon, K. D., Wood, R., Heppner, M. J., \& Gysbers, N. C. (2007). A clusteranalytic investigation of subtypes of adult career counseling clients: Toward a taxonomy of career problems. Journal of Career Assessment, 15(1), 6686.

Murphy, G. C., \& Athanasou, J. A. (1999). The effect of unemployment on mental health. Journal of Occupational and Organizational Psychology, 72(1), 8399.

Natkin, S. (2006). Video games and interactive media: A glimpse at new digital entertainment. Video games and Interactive Media: A Glimpse at New 


\section{Digital Entertainment, 135.}

Parent, G., \& Sautory, O. (2015). L'accompagnement des demandeurs d'emploi. Travail et emploi, (3), 75-89.

Paul, K. I., \& Moser, K. (2009). Unemployment impairs mental health: Metaanalyses. Journal of Vocational Behavior, 74(3), 264-282.

Piaget, J. (1945). La formation du symbole chez l'enfant: Imitation, jeu et rêve, image et représentation [Play, dreams and imitation in

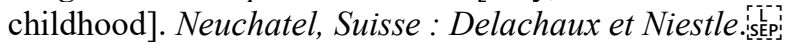

Pochic, S., \& Bory, A. (2014). Expériences de pertes d'emploi: la crise vue d'en bas. Travail et emploi, (2), 5-18.

Postle, D. (1993). Putting the heart back into learning. Using experience for learning, 33-45.

Roques, M. (2008). Les dimensions psychologiques et psychosociales dans l'évaluation de l'efficacité des dispositifs d'insertion. Pratiques psychologiques, 14(3), 375-388.

Rosenberg, M. (1965). Society and the adolescent self-image. Princeton, NJ: Princeton University Press.

Rotter, J. B. (1966). Generalized expectancies for internal versus external control of reinforcement. Psychological monographs: General and applied, 80(1), 1.

Rotter, J. B. (1990). Internal versus external control of reinforcement: A case history of a variable. American Psychologist, 45(4), 489.

Ryan, R. M., Rigby, C. S., \& Przybylski, A. (2006). The motivational pull of video games: A self-determination theory approach. Motivation and Emotion, 30(4), 344-360.

Salognon, M. (2006). Modes de jugement des entreprises et insertion des chômeurs de longue durée. Observation de la méthode IOD. Travailler pour être intégré? Mutations des relations entre emploi et protection sociale, 163179.

Sanchez, E., Ney, M., \& Labat, J. M. (2011). Jeux sérieux et pédagogie universitaire: de la conception à l'évaluation des apprentissages. Revue internationale des technologies en pédagogie universitaire, 8(1-2), 48-57.

Simões, J., Redondo, R. D., \& Vilas, A. F. (2013). A social gamification framework for a K-6 learning platform. Computers in Human Behavior, 29(2), 345353.

Strommen, E. F., \& Lincoln, B. (1992). Constructivism, technology, and the future of classroom learning. Education and Urban Society, 24(4), 466-476.

Vinokur, A. D., Van Ryn, M., Gramlich, E. M., \& Price, R. H. (1991). Long-term follow-up and benefit-cost analysis of the Jobs Program: a preventive intervention for the unemployed. Journal of Applied Psychology, 76(2), 213.

Vygotsky, L. S. (1978). Mind in society: The development of higher psychological processes. (M. Cole, V. John-Steiner, S. Scribner, \& E. Souberman, Eds.). Cambridge, Massachusetts: Harvard University Press. 
Wanberg, C. R., Watt, J. D., \& Rumsey, D. J. (1996). Individuals without jobs: An empirical study of job-seeking behavior and reemployment. Journal of Applied Psychology, 81(1), 76.

Wanberg, C. R., \& Banas, J. T. (2000). Predictors and outcomes of openness to changes in a reorganizing workplace. Journal of Applied Psychology, 85(1), 132.

Winnicott, D. W., \& Monod, C. (1975). Jeu et réalité: l'espace potentiel (Vol. 1). Paris: Gallimard.

Witmer, B. G., \& Singer, M. J. (1998). Measuring presence in virtual environments: A presence questionnaire. Presence: Teleoperators and Virtual Environments, 7(3), 225-240.

Wouters, P., Van Nimwegen, C., Van Oostendorp, H., \& Van Der Spek, E. D. (2013). A meta-analysis of the cognitive and motivational effects of serious games. Journal of Educational Psychology, 105(2), 249.

Zyda, M. (2005). From visual simulation to virtual reality to games. Computer, 38(9), 25-32. 